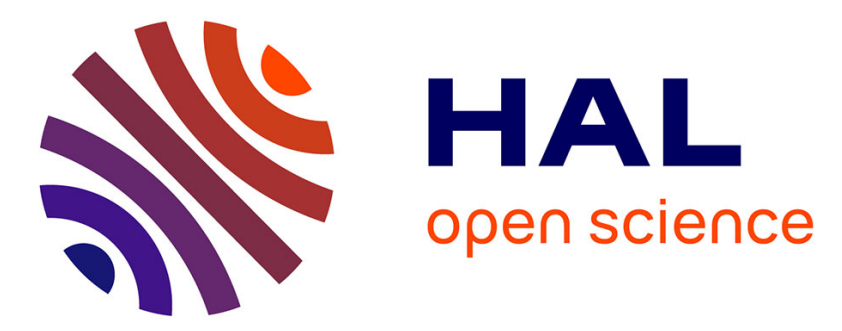

\title{
Spreading Factor Allocation Strategy for LoRa Networks under Imperfect Orthogonality
}

Licia Amichi, Megumi Kaneko, Nancy El Rachkidy, Alexandre Guitton

\section{To cite this version:}

Licia Amichi, Megumi Kaneko, Nancy El Rachkidy, Alexandre Guitton. Spreading Factor Allocation Strategy for LoRa Networks under Imperfect Orthogonality. IEEE International Conference on Communications, May 2019, Shanghai, China. hal-02022622

\section{HAL Id: hal-02022622 \\ https://hal.uca.fr/hal-02022622}

Submitted on 18 Feb 2019

HAL is a multi-disciplinary open access archive for the deposit and dissemination of scientific research documents, whether they are published or not. The documents may come from teaching and research institutions in France or abroad, or from public or private research centers.
L'archive ouverte pluridisciplinaire HAL, est destinée au dépôt et à la diffusion de documents scientifiques de niveau recherche, publiés ou non, émanant des établissements d'enseignement et de recherche français ou étrangers, des laboratoires publics ou privés. 


\title{
Spreading Factor Allocation Strategy for LoRa Networks under Imperfect Orthogonality
}

\author{
Licia Amichi*, Megumi Kaneko*, Nancy El Rachkidy ${ }^{\dagger}$, and Alexandre Guitton ${ }^{\dagger}$ \\ * National Institute of Informatics, 2-1-2 Hitotsubashi, Chiyoda-ku, 101-8430 Tokyo, Japan \\ $\dagger$ Université Clermont Auvergne, CNRS, LIMOS, F-63000 Clermont-Ferrand, France
}

Email: licia.amichi@etu.upmc.fr, megkaneko@nii.ac.jp, \{nancy.el_rachkidy, alexandre.guitton\}@uca.fr

\begin{abstract}
Low-Power Wide-Area Network (LPWAN) based on LoRa physical layer is envisioned as one of the most promising technologies to support future Internet of Things (IoT) systems. LoRa provides flexible adaptations of coverage and data rates by allocating different Spreading Factors (SFs) to end-devices. Although most works so far had considered perfect orthogonality among SFs, the harmful effects of inter-SF interferences have been demonstrated recently. Therefore in this work, we consider the problem of SF allocation optimization under co-SF and interSF interferences, for uplink transmissions from end-devices to the gateway. To provide fairness, we formulate the problem as maximizing the minimum achievable average rate in LoRa, and propose a SF allocation algorithm based on matching theory. Numerical results show that our proposed algorithm enables to jointly enhance the minimal user rates, network throughput and fairness, compared to baseline SF allocation methods.
\end{abstract}

Index Terms-LoRa, Spreading Factor, Imperfect Orthogonality, Resource Allocation Optimization, Matching Theory

\section{INTRODUCTION}

Tens of billions of Internet of Thing (IoT) devices are expected by 2020 , for enabling groundbreaking applications such as smart cities, intelligent transportation systems or harsh environment monitoring. Many of these IoT Wireless Sensor Networks (WSNs) will strongly rely on Low-Power Wide-Area Network (LPWAN) technologies such as Long Range (LoRa) [1,2]. LoRa is one of the most prominent physical layer technologies for LPWAN, as it operates in the ISM unlicensed bands and enables flexible adaptations of transmission rates and coverages under low energy consumption [3]. LoRa is based on Chirp Spread Spectrum (CSS) modulation, where each chirp encodes $2^{m}$ symbol values, for Spreading Factor (SF) $m$. On top of LoRa physical layer, LoRaWAN defines the MAC layer protocol standardized by LoRa Alliance [4]. The LoRaWAN architecture is a star-like topology, where end-devices communicate with gateways over several channels based on ALOHA mechanism, with duty cycle limitations [1]. In LoRaWAN, six SFs ranging from $m=7$ to 12 are used, where smaller SFs provide higher data rates but reduced ranges, while larger SFs allow longer ranges but lower rates [2].

One of the main issues in LoRa and LoRaWAN is the throughput limitation: the physical bitrate varies between 300 and 50000 bps [4]. In addition, collisions are very harmful to the system performance as the LoRa gateway is unable to

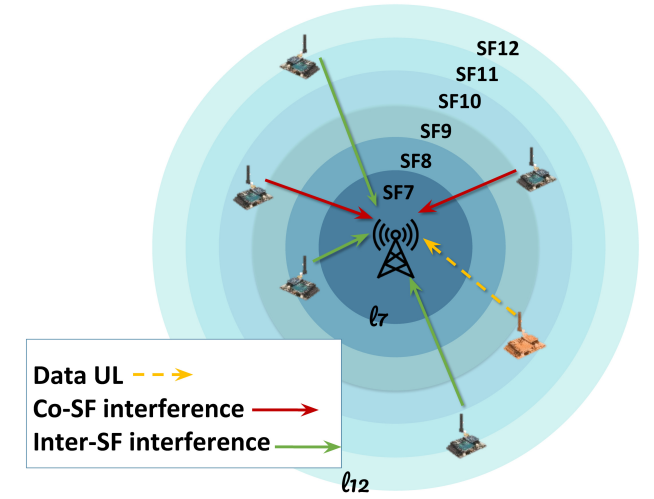

Fig. 1. LoRa system, with end-devices transmitting simultaneously

correctly decode simultaneous signals sent by devices using the same SF and the same channel. Such interferences will be referred to as co-SF interferences. Although SFs were widely believed to be orthogonal among themselves, some recent studies have shown that this is not the case by experimentally evaluating the effects of inter-SF interferences [5, 6]. Thus, authors in [7] have analyzed the achievable uplink LoRa throughput under imperfect SF orthogonality, and have demonstrated the harmful impact of both co-SF and inter-SF interferences on the overall throughput.

In order to improve the LoRa system performance, a number of works have proposed resource optimization methods [810]. However, most papers so far have assumed a perfect orthogonality among SFs. In particular, the authors in [8] design a channel and power allocation algorithm, that maximizes the minimal rate. However, the allocated channels are perfectly orthogonal, and no SF allocation nor SF-dependent rates were not considered, despite the strong dependency of the rate to SFs. In addition, the solution of [8] requires instantaneous Channel State Information (CSI) feedback, which is not adapted to LoRa networks due to their energy consumption limitations [4]. In [9], a heuristic SF-allocation is proposed, where users with similar path losses are simply assigned to the same channel, then to each SF according to their distance to the gateway. Although the issue of inter-SF interferences was highlighted, it was ignored in their proposed solution. In [10], a method for decoding superposed LoRa signals using 
the same SF was proposed.

Therefore, in this work, we consider the issue of SF allocation optimization under both co-SF and inter-SF interferences. In particular, we focus on the problem of maximizing the minimum achievable short-term average rate in the uplink, whereby short-term average rate is defined as the average rate over random channel fadings, but given a fixed position of enddevices. This metric is especially suited for LoRa networks, since the end-devices will likely be fixed for a certain period of time (at least for a few seconds) in many applications, and their positions known at the gateway, as in the conventional signal-strength-based SF allocation method [4]. Our focus here is the max-min fairness in terms of throughput, without taking into consideration the energy of end-devices. As LoRaWAN enforces a limited duty-cycle, the energy consumption of each end-devices remains limited. Firstly, we formulate our optimization problem by modeling the achievable uplink shortterm average rate under co-SF and inter-SF interferences. Next, given the mathematical intractability of the optimization problem, we propose an SF-allocation algorithm based on matching theory. We show its stability and convergence properties, and analyze its computational complexity. Finally, numerical results demonstrate that, compared to baseline SFallocation schemes, our proposed method not only provides larger minimum rates, but also jointly improves the network throughput and fairness level.

\section{SySTEM MODEL}

We consider an uplink LoRa system with a single gateway located at the center of a cell of radius $R \mathrm{~km}$ and $N$ enddevices uniformly distributed and simultaneously active as depicted in Figure 1. End-devices transmit on the same channel $c$ of bandwidth $B W$, with a power $P_{\max }$ and a duty cycle of $100 \%$. We denote the set of SFs by $\mathcal{M}=\{7,8, \ldots, 12\}$, where $M=|\mathcal{M}|=6$. Each $\mathrm{SF}_{m}, m \in \mathcal{M}$, has a data bit-rate equal to $R_{m}$ [3],

$$
R_{m}=\frac{m \times C R}{\frac{2^{m}}{B W}}
$$

where the Coding Rate is given in [3] as $C R=\frac{4}{4+n}$ with $n \in\{1,2,3,4\}$.

The uplink instantaneous Signal-to-Noise Ratio (SNR), $\gamma_{n m}$, for end-device $n$ at $\mathrm{SF}_{m}$ is given by [7],

$$
\gamma_{n m}=\frac{P_{\max }\left|h_{n}\right|^{2} \mathrm{~A}\left(f_{c}\right)}{r_{n}^{\alpha} \sigma_{c}^{2}},
$$

where $h_{n}$ is the channel gain between $n$ and the gateway, $\mathrm{A}\left(f_{c}\right)=\left(f_{c}^{2} \times 10^{-2.8}\right)^{-1}$ is the deterministic loss, $f_{c}$ is the carrier frequency, $r_{n}$ the distance from enddevice $n$ to the gateway, $\alpha$ the path loss exponent and $\sigma_{c}^{2}=-174+\mathrm{NF}+10 \log (B W)[\mathrm{dBm}]$ is the Additive White Gaussian Noise (AWGN) where NF is the receiver Noise Figure. Assuming Rayleigh fading channels, the SNR $\gamma_{n m}$ is modeled as an exponential random variable with mean $\bar{\gamma}_{n m}=\frac{\mathrm{A}\left(f_{c}\right) P_{\max }}{r_{n}^{\alpha} \sigma_{c}^{2}}$.

\begin{tabular}{|c|c|c|c|c|c|}
\hline $\begin{array}{c}\text { SF } \\
m\end{array}$ & $\begin{array}{c}\text { Bit-rate } \\
{[\mathrm{kb} / \mathrm{s}]}\end{array}$ & $\begin{array}{c}\text { Receiver } \\
\text { sensitiv- } \\
\text { ity [3] } \\
{[\mathrm{dBm}]}\end{array}$ & $\begin{array}{c}\text { Reception } \\
\text { thresh. } \\
\theta_{\mathrm{rx} m}[\mathrm{~dB}]\end{array}$ & $\begin{array}{c}\text { InterSF } \\
\text { thresh. [11] }\end{array}$ & $\begin{array}{c}\text { Distance } \\
\tilde{\theta}_{m}[\mathrm{~dB}]\end{array}$ \\
\hline $\mathbf{r a n g e s}$ \\
\hline 8 & 5.47 & -123 & -6 & -7.5 & $0-l_{7}$ \\
\hline 9 & 3.13 & -126 & -9 & -9 & $l_{7}-l_{8}$ \\
\hline 10 & 1.76 & -129 & -12 & -13.5 & $l_{8}-l_{9}$ \\
\hline 11 & 0.98 & -132 & -15 & -15 & $l_{9}-l_{10}$ \\
\hline 12 & 0.54 & -134.5 & -17.5 & -18 & $l_{10}-l_{11}$ \\
\hline
\end{tabular}

LORA CHARACTERISTICS AT $B W=125 \mathrm{KHz}$ [7]

The area covered by each SF is given by the distance ranges in Table I with [7],

$$
l_{m}=\mathrm{e}^{\frac{1}{\alpha} \times \ln \left(\frac{\mathrm{A}\left(f_{c}\right)}{L B_{m}}\right)},
$$

where $L B_{m}$ is the link budget of the $\mathrm{SF}_{m}$ defined as $L B_{m}=$ $P_{\max }-\theta_{r x_{m}}$, given the receiver sensitivity of each $\mathrm{SF}_{m} \theta_{r x_{m}}$ in Table I. Hence, larger SFs result in larger communication ranges, with $l_{12}=R$.

If there is only one device $n$ assigned to $\mathrm{SF}_{m}$, this device is only subject to inter-SF interferences caused by devices using a different SF. Hence the inter-SF Signal-to-Interference-plusNoise-Ratio (SINR) of end-device $n$ can be expressed as

$$
\operatorname{SINR}_{n m}^{\text {inter }}=\frac{\gamma_{n m}}{\sum_{i \in \mathcal{N}_{-n}} \sum_{j \in \mathcal{M}_{-m}} s_{i j} \gamma_{i j}+1},
$$

where $\mathcal{N}_{-n}=\mathcal{N} \backslash\{n\}, \mathcal{M}_{-m}=\mathcal{M} \backslash\{m\}$ and $s_{i j}$ is a binary variable used to indicate whether $n$ uses $m$,

$$
s_{i j}=\left\{\begin{array}{l}
1, \text { if } i \text { uses } j \\
0, \text { otherwise. }
\end{array}\right.
$$

When there is more than one device assigned to a SF, these devices are subject to both inter-SF and co-SF interferences. However, as shown in [5-7], co-SF interferences are largely dominant over inter-SF interferences ${ }^{1}$. Therefore, the co-SF SINR of end-device $n$ on $\mathrm{SF}_{m}$ is written as,

$$
\operatorname{SINR}_{n m}^{\mathrm{co}}=\frac{\gamma_{n m}}{\sum_{i \in \mathcal{N}_{-n}} s_{i m} \gamma_{i m}+1} .
$$

In conformity to LoRaWAN standards, instantaneous CSI feedback is not assumed, unlike [8]. Hence, the SF allocation is performed every period of time, during which the long-term fading instance, i.e., path loss distance, can be assumed to be fixed. Therefore, the achievable uplink short-term average rate for device $n$ at $\mathrm{SF}_{m}$ is given similarly to [7] by,

$$
\tau_{n m}=R_{m} \times P_{\text {cap }}^{(n, m)},
$$

where $R_{m}$ is the data bit-rate of the $\mathrm{SF}_{m}$ and $P_{\text {cap }}^{(n, m)}$ the probability of successful transmission, which is analyzed in the following section.

\footnotetext{
${ }^{1}$ LoRa chirps were especially designed to yield limited inter-SF interferences.
} 


\section{PROBLEM FORMULATION}

In this section, we formulate the considered optimization problem. Since fairness will be crucial in LoRa systems, we strive to maximize the minimal uplink short-term average rate over devices and SFs, under co-SF and inter-SF interferences. We first derive the expression of $P_{\text {cap }}^{(n, m)}$. Assuming $N>1$, there are two cases:

1) One device $n$ at $\mathrm{SF}_{m}$ : device $n$ is only subject to inter-SF interferences. The transmission can be successfully decoded if the node satisfies the inter-SF as well as the reception condition. In this case inter-SF interferences are more critical than the reception condition since there will always be inter-SF interferences for $N>1$. Hence the probability of successful transmission can be written as,

$$
P_{\text {cap }_{\mathrm{iSF}}}^{(n, m)}=P\left(\operatorname{SINR}_{n m}^{\mathrm{inter}} \geq \tilde{\theta}_{m}\right),
$$

where $\operatorname{SINR}_{n m}^{\text {inter }}$ is given in (4) and $\tilde{\theta}_{m}$ is the inter-SF interference capture threshold for $\mathrm{SF}_{m}$, defined in Table I. Using the random instantaneous SNR variables $\gamma_{n m}$ for all $(n, m)$ and marginalizing over them, we show in the Appendix with similar calculations as in [7] that (7) can be written as,

$$
P_{\text {cap }_{\mathrm{iSF}}}^{(n, m)}=\mathrm{e}^{-\frac{\tilde{\theta}_{m} \sigma_{c}^{2} r_{n}^{\alpha}}{\mathrm{A}\left(f_{c}\right) P_{\max }}} \prod_{\substack{(i, j) \in \\ \mathcal{N}_{-n} \times \mathcal{M}_{-m}}} \frac{1}{\tilde{\theta}_{m} s_{i j}\left(\frac{r_{n}}{r_{i}}\right)^{\alpha}+1} .
$$

2) More than one device at $\mathrm{SF}_{m}$ : in this case, co-SF interferences largely dominate the inter-SF interferences as well as reception condition as explained in Section II. Therefore, the success probability is expressed as in [12],

$$
P_{\mathrm{cap}_{\mathrm{coSF}}}^{(n, m)}=P\left(\mathrm{SINR}_{n m}^{\mathrm{co}} \geq \theta_{\mathrm{co}}\right) \text {, }
$$

where $\operatorname{SINR}_{n m}^{\mathrm{co}}$ is given in (5) and $\theta_{\text {co }}$ is the co-SF capture threshold which is equal to $6 \mathrm{~dB}$ for all $\mathrm{SF}_{m}$ [3,12]. With similar calculations as in the Appendix, we obtain

$$
P_{\text {cap }_{\operatorname{cosF}}}^{(n, m)}=\mathrm{e}^{-\frac{\theta_{\mathrm{co} \sigma_{c}^{2} r_{n}^{\alpha}}}{A\left(f_{c}\right) P_{\max }}} \prod_{i \in \mathcal{N}_{-n}} \frac{1}{\theta_{\mathrm{co}} s_{i m} \times\left(\frac{r_{n}}{r_{i}}\right)^{\alpha}+1} .
$$

Given the above analysis, we can now formulate our objective function as follows (for $N>1$ ),

$$
\max _{s_{n m}} \min _{\substack{(n, m) \in \\ \mathcal{N} \times \mathcal{M} \\ \text { s.t. } s_{n m} \neq 0}} s_{n m} R_{m} P_{\text {cap }}^{(n, m)}
$$

where the minimization is over the $s_{n m}$ that are non-zero, and

$$
P_{\mathrm{cap}}^{(n, m)}=I\left(\sum_{k \in \mathcal{N}} s_{k m} \geq 2\right) P_{\mathrm{cap}_{\mathrm{coSF}}}^{(n, m)}+I\left(\sum_{k \in \mathcal{N}} s_{k m}=1\right) P_{\mathrm{cap}}^{(n, m)},
$$

where $I(C)$ is the indicator function, i.e., it equals 1 if the condition $C$ is verified and 0 otherwise.
Finally, the overall optimization problem becomes

$$
\begin{aligned}
& (P) \max _{s_{n m}} \min _{\substack{(n, m) \in \in \\
\mathcal{N} \times \mathcal{M} \\
\text { s.t.s } s_{n m} \neq 0}} s_{n m} R_{m}\left[I\left(\sum_{k \in \mathcal{N}} s_{k m} \geq 2\right) \mathrm{e}^{-\frac{\theta_{\mathrm{co}} \sigma_{c}^{2} r_{n}^{\alpha}}{\mathrm{A}\left(f_{c}\right) P_{\max }}}\right. \\
& \times \prod_{i \in \mathcal{N}_{-n}} \frac{1}{\theta_{\operatorname{co}} s_{i m}\left(\frac{r_{n}}{r_{i}}\right)^{\alpha}+1}+I\left(\sum_{k \in \mathcal{N}} s_{k m}=1\right) \mathrm{e}^{-\frac{\tilde{\theta}_{m} \sigma_{c}^{2} r_{n}^{\alpha}}{\mathrm{A}\left(f_{c}\right) P_{\max }}} \\
& \left.\times \prod_{\substack{(i, j) \in \\
\mathcal{N}_{-n} \times \mathcal{M}}} \frac{1}{\tilde{\theta}_{m} s_{i j}\left(\frac{r_{n}}{r_{i}}\right)^{\alpha}+1}\right] \\
& \text { s.t. } \\
& \text { C1: } s_{n m} \in\{0,1\} \quad \forall(n, m) \in \mathcal{N} \times \mathcal{M} \\
& \text { C2: } \sum_{m \in \mathcal{M}} s_{n m} \leq 1, \forall n \in \mathcal{N} \\
& \text { C3: } \sum_{n \in \mathcal{N}} s_{n m} \leq \mathrm{N}_{\max }(m), \forall m \in \mathcal{M} \\
& \text { C4: if } N>M, 1 \leq \sum_{n \in \mathcal{M}} s_{n m}, \forall m
\end{aligned}
$$

This optimization problem is constrained by the binary allocation variable $s_{n m} \in\{0,1\}$ (13a), the fact that an enddevice $n$ is assigned to at most one SF (13b) and that the maximal number of devices at $\mathrm{SF}_{m}$ is upper bounded by $\mathrm{N}_{\max }(m)(13 \mathrm{c})^{2}$, if there are enough devices $(N>M)$, no SFs should remain unused (13d). $(P)$ is hence an integer programming problem, given the binary variables $s_{n m}$, with a non-linear objective function. This is an NP-hard problem [13], hence it is difficult to obtain its optimal solution directly. Therefore, we propose an optimized SF allocation method, using tools from matching theory.

\section{Proposed Method}

Matching theory is a promising tool for resource allocation in wireless networks [14]. According to this theory, our considered allocation problem $(P)$ can be classified as a manyto-one matching problem with conventional externalities and peer effects. There are two sets of players, the set of SFs and the set of end-devices, where each element seeks to be matched with players of the opposing set. A device will prefer to be matched to the SF offering the highest utility, while each SF prefers to be matched with the group of devices with the highest utility. The difficulty of our problem is that there is an interdependence between nodes' preferences, i.e., whenever a device is matched to an SF, the preferences of the other devices may change due to co-SF interferences. In addition to these conventional externalities (preference interdependence) and unlike the problem in [8] where only orthogonal channels (not $\mathrm{SFs}$ ) were considered, our problem exhibits peer effects that are caused by inter-SF interferences. That is, the preferences of a device depend not only on the identity of the SF and the number of devices assigned to it, but also on the assignment of devices to other SFs (since they cause inter-SF interferences). Hence, to solve $(P)$, we propose a many-to-one matching

\footnotetext{
${ }^{2}$ Setting $\mathrm{N}_{\max }(m)$ enables to control the harmful effects of co-SF interferences, and reduces the computational complexity of the proposed method, as shown in Sections IV-B and V-C.
} 
algorithm between the set of SFs $\mathcal{M}$ and the set of end-devices $\mathcal{N}$. First, we define the basic concepts of matching theory.

Matching pair: a couple $(n, m)$ assigned to each other.

Quotas of a player: the maximum number of players with which it can be matched

- Each end-device has a quota of 1 (13b),

- Each $\mathrm{SF}_{m}$ has a quota of $\mathrm{N}_{\max }(m)$ devices (13c).

Utility of a node: defined for our problem as its short-term average rate. If it is the only device at $\mathrm{SF}_{m}$,

$$
U_{n}=R_{m} \mathrm{e}^{-\frac{\tilde{\theta}_{m} \sigma_{c}^{2} r_{n}^{\alpha}}{\mathrm{A}\left(f_{c}\right) P_{\max }}} \prod_{\substack{(i, j) \in \\ \mathcal{N}_{-n} \times \mathcal{M}_{-m}}} \frac{1}{\tilde{\theta}_{m} s_{i j}\left(\frac{r_{n}}{r_{i}}\right)^{\alpha}+1} .
$$

If it shares the $\mathrm{SF}_{m}$ with other devices,

$$
U_{n}=R_{m} \mathrm{e}^{-\frac{\theta_{c \mathrm{co}} \sigma_{c}^{2} r_{n}^{\alpha}}{\mathrm{A}\left(f_{c}\right) P_{\max }}} \prod_{i \in \mathcal{N}_{-n}} \frac{1}{\theta_{\mathrm{co}} s_{i m}\left(\frac{r_{n}}{r_{i}}\right)^{\alpha}+1} .
$$

Utility of an SF: defined for our problem as the minimum short-term average rate among the devices assigned to it. If $\mathrm{SF}_{m}$ is matched to one device only:

$$
U_{m}=\min _{n \in \mathcal{N}_{m}^{*}} R_{m} \mathrm{e}^{-\frac{\tilde{\theta}_{m} \sigma_{c}^{2} r_{n}^{\alpha}}{\mathrm{A}\left(f_{c}\right) P_{\max }}} \prod_{\substack{(i, j) \in \\ \mathcal{N}_{-n} \times \mathcal{M}_{-m}}} \frac{1}{\tilde{\theta}_{m} s_{i j}\left(\frac{r_{n}}{r_{i}}\right)^{\alpha}+1},
$$

where $\mathcal{N}^{*}{ }_{m}$ is the set of devices assigned to the $\mathrm{SF}_{m}$, otherwise $U_{m}$ is given as

$$
U_{m}=\min _{n \in \mathcal{N}_{m}^{*}} R_{m} \mathrm{e}^{-\frac{\theta_{\mathrm{co}} \sigma_{r}^{2} r_{n}^{\alpha}}{\mathrm{A}\left(f_{c}\right) P_{\max }}} \prod_{i \in \mathcal{N}_{-n}} \frac{1}{\theta_{\mathrm{co}} s_{i m}\left(\frac{r_{n}}{r_{i}}\right)^{\alpha}+1} .
$$

Preference relation: a player $q$ prefers a player $p_{1}$ over the player $p_{2}$, if the utility of $q$ is higher when it is matched to $p_{1}$ than when it is matched to $p_{2}$.

Blocking pair: a matching pair $(n, m)$ is a blocking pair when $U_{n}$ or $U_{m}$ is higher when $n$ uses $m$, than when they use their current matches, without lowering the utilities of any other device nor SF. In this case, $n$ will leave its current match to be matched to $m$.

Two-sided stable matching: a matching solution where there is no blocking pair.

\section{A. Proposed algorithm}

In this subsection, we describe the steps of the proposed matching-based algorithm which exploits matching techniques as in $[8,14]$, tailored to our specific problem. First, the scheduler located at the gateway performs an initial matching between the set of SFs $M$ and the set of end-devices $N$ by the Initial Matching in Algorithm 1. Next, it swaps the matching pairs obtained in the previous step until reaching a two-sided exchange stable matching by Matching Refinement in Algorithm 2. Details of these steps are given below.

Let $\mathcal{L}_{U}$ denote the set of end-devices that are not allocated to any $\mathrm{SF}, \mathrm{req}_{m}$ the requests received by the $\mathrm{SF}_{m}$, and $\mathcal{A}_{m}$ the set of devices assigned to the $\mathrm{SF}_{m}$. We suppose that the gateway knows the distance of all end-devices to it.
Initialization: the gateway starts by initializing the preference lists of end-devices and SFs. Each device $n$ with a distance $r_{n}$ to the gateway, can only use SFs including it in their coverage area $\left(r_{n} \leq l_{m}\right)$, therefore,

$$
\mathcal{L}_{p, n}=\left\{m \in \mathcal{M}, \text { st } r_{n} \leq l_{m}\right\},
$$

$\mathcal{L}_{p, n}$ is arranged according to the increasing order of the distance threshold of the SFs $\left(l_{m}, m \in M\right)$, i.e, an SF with higher achievable rate is preferred. On the other hand, an $\mathrm{SF}_{m}$ only considers devices having a distance to the gateway lower than $l_{m}$,

$$
\mathcal{L}_{p, m}=\left\{n \in \mathcal{N}, \text { st } r_{n} \leq l_{m}\right\} .
$$

$\mathcal{L}_{p, m}$ is ordered such that, a user $n_{1} \in \mathcal{L}_{p, m}$ is ranked before another user $n_{2} \in \mathcal{L}_{p, m}$ if $n_{1}$ is located in the ring of $\mathrm{SF}_{m}$ $\left(n_{1} \in\left(l_{m-1}, l_{m}\right]\right)$ but not $n_{2}\left(n_{2} \notin\left(l_{m-1}, l_{m}\right]\right)$, or both are in the ring of $\mathrm{SF}_{m}$ but $n_{1}$ is closer to the gateway than $n_{2}$ $\left(\left|l_{m-1}-r_{n_{1}}\right|<\left|l_{m-1}-r_{n_{2}}\right|\right)$.

Unmatched end-devices are added to $\mathcal{L}_{U}$.

Initial Matching: for each device $n$ in the unmatched list $\mathcal{L}_{U}$, if $\mathcal{L}_{p, n} \neq \emptyset, n$ requests its first preferred $\mathrm{SF}$ and removes it from $\mathcal{L}_{p, n}$, otherwise the device is removed from $\mathcal{L}_{U}$ since all SFs it can use have already reached their quota. Then, each $\mathrm{SF}_{m}$ either accepts all current requests if its quota allows it, or it accepts the requests of its most preferred devices that fulfill its quota, if not. This process is repeated until $\mathcal{L}_{U}$ becomes empty. Note that, setting this quota of $N_{\max }(m)$ devices for each $\mathrm{SF}_{m}$ also avoids large contention delays.

Matching Refinement: for each matching pair $(n, m)$ the algorithm calculates $U_{m}$ using (16) if it is only assigned to device $n$ and (17) in the other case. The utility of device $n$ is calculated by (14) if it is the only one at $\mathrm{SF}_{m}$, and with (15) otherwise. Firstly, if there is an $\mathrm{SF}_{l}$ that is not assigned to any device that allows increasing $U_{n}$, the device leaves $\mathrm{SF}_{m}$ to be matched with $\mathrm{SF}_{l}$. Then, the algorithm calculates the utilities of every pair $(k, l)$, and makes a swap between $(n, m)$ and $(k, l)$ and determines their new utilities. If $(k, m)$ or $(n, l)$ is a blocking pair the algorithm validates the swap. This swapping step is repeated until reaching a two-sided stable matching.

\section{B. Algorithm analysis}

We now prove the stability and convergence of the proposed algorithm, and analyze its computational complexity.

Proposition 1. Stability: When the proposed algorithm terminates, it finds a two-sided exchange stable matching.

Proof. Let's assume that the proposed algorithm terminates and the final matching is not two-sided exchange stable. Then, the matching contains at least one more blocking pair $(k, m)$ or $(n, l)$ where the utility of at least one player among $\{n, m, k, l\}$, can be improved without lowering others' utility. Accordingly, the proposed algorithm did not terminate and will continue, thereby the matching is not final, which contradicts the initial assumption. 

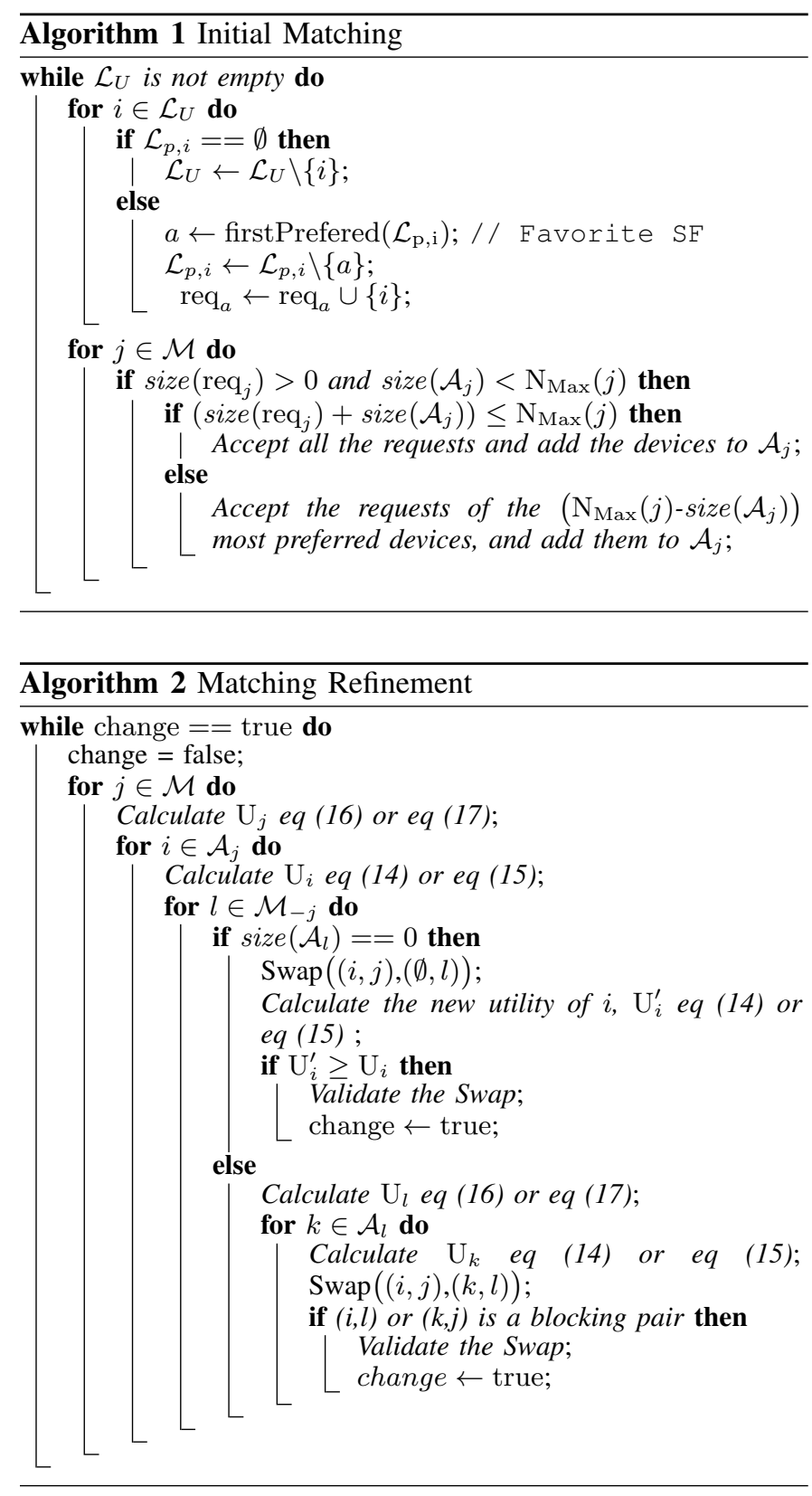

Proposition 2. Convergence: After a finite number of swap operations, the algorithm eventually converges to a two-sided exchange stable matching.

Proof. A swap operation occurs if it improves the utility of at least one player without decreasing the others', hence the utilities can only rise. Additionally, the maximal throughput that can be achieved on an $\mathrm{SF}_{m}$ is upper-bounded by the data bit-rate $R_{m}$, meaning that each $\mathrm{SF}_{m}$ and the devices assigned to it have utilities upper bounded by $R_{m}$.

The number of potential swap operations is finite: device assigned to $\mathrm{SF}_{l}$ can make at most $\mathrm{N}_{\max }(l) \times \sum_{j \in \mathcal{M}_{-l}} \mathrm{~N}_{\max }(j)$ swap operations. The total number of swap operations is thus upper-bounded by $\sum_{l \in \mathcal{M}} \mathrm{N}_{\max }(l) \times \sum_{j \in \mathcal{M}_{-l}} \mathrm{~N}_{\max }(j)$.
Proposition 3. Complexity: The running time of our proposed algorithm is upper-bounded by $\mathcal{O}\left(N M+Q^{2} M^{2}\right)$.

Proof. Initial matching complexity: in the worst case, all the devices have the same preference list, and they are located in the area covered by all the SFs. At round ${ }_{1}$ the gateway receives $N$ requests, at round 2 it receives $N-\mathrm{N}_{\max }\left(m_{1}\right)$ requests, at $\operatorname{round}_{i}$ it receives $N-\sum_{k=1}^{M-1} \mathrm{~N}_{\max }\left(m_{i}\right)$ requests. Therefore, the total number of requests equals $N M-\sum_{i=1}^{M-1}(M-i) \times$ $\mathrm{N}_{\max }\left(m_{i}\right)$. The complexity of the initial matching is upper bounded by: $\mathcal{O}(N M)$.

Matching refinement complexity: in each iteration, for each $\mathrm{SF}_{m}$, the algorithm considers at most $\mathrm{N}_{\max }(m)$ devices and examines $\sum_{l \in \mathcal{M}_{-m}} N_{\max }(l)$ swap operations for each of these devices. Therefore, the number of swap operations that are examined in one iteration is upper bounded by $\sum_{m \in \mathcal{M}} \mathrm{N}_{\max }(m) \times \sum_{l \in \mathcal{M}-m} \mathrm{~N}_{\max }(l)$. Let $Q=\max _{m \in \mathcal{M}}\left\{\mathrm{N}_{\max }(m)\right\}$, thus the computational complexity of the matching refinement is upper bounded by $\mathcal{O}\left(Q^{2} M(M-1)\right)$.

In summary, the computational complexity of our algorithm is upper bounded by $\mathcal{O}\left(N M+Q^{2} M^{2}\right)$.

Note that this complexity is not excessive as our algorithm is run at the gateway which is not computationally-limited.

\section{Numerical Results}

\section{A. Simulation Settings}

We consider a cell of radius $\mathrm{R}=1 \mathrm{~km}$ [12], with a varying number of devices $N$ from $\mathrm{N}=2$ to 40 . Note that all devices transmit with a duty cycle of $100 \%$. Hence, with a duty cycle of $1 \%$ as preconized in LoRaWAN [1], the actual number of end-devices would be 100 -fold ${ }^{3}$, i.e., up to 4000 . All end-devices transmit in the channel of carrier frequency $f_{c}=868[\mathrm{MHz}]$ with a bandwidth $B W=125[\mathrm{kHz}]$ and power $P_{\max }=14[\mathrm{dBm}]$. We consider a lossy urban environment, therefore we take a path loss exponent equal to 4 as in [7,12]. The computer simulation environment has been implemented in Matlab ${ }^{4}$.

\section{B. Baseline schemes}

We consider two baseline schemes for performance comparison the random SF allocation [7], and the distance-SF allocation algorithms [11], with a maximal number of simultaneously transmitting devices equal to $A$ (specified later):

Random SF: the gateway chooses randomly $A$ devices among $\mathcal{N}$ and assigns a random SF to each of these devices among the possible SFs.

Distance SF: the gateway chooses randomly $A$ devices among $\mathcal{N}$. Then, the SF for each of these devices is determined by

\footnotetext{
${ }^{3}$ The evaluations are made for $100 \%$ duty cycle as this is the most challenging case. Hence, much better performance can be expected in the case of $1 \%$ duty cycle.

${ }^{4}$ All results are based on our mathematical model detailed in the Appendix and whose validity has been proved in [7].
} 


\begin{tabular}{|c|c|c|c|c|c|c|}
\hline$N_{\operatorname{Max}}$ & SF7 & SF8 & SF9 & SF10 & SF11 & SF12 \\
\hline 1 & 4.82 & 1.51 & 1.06 & $4.7 \mathrm{e}-1$ & $2.7 \mathrm{e}-1$ & $1.9 \mathrm{e}-1$ \\
\hline 2 & $7.7 \mathrm{e}-2$ & $1.1 \mathrm{e}-7$ & $9.3 \mathrm{e}-14$ & $7.8 \mathrm{e}-25$ & $6.7 \mathrm{e}-46$ & $3.7 \mathrm{e}-78$ \\
\hline 3 & $2.7 \mathrm{e}-3$ & $8.2 \mathrm{e}-9$ & $2 \mathrm{e}-15$ & $8.2 \mathrm{e}-27$ & $3.1 \mathrm{e}-49$ & $1.3 \mathrm{e}-84$ \\
\hline 4 & $9.9 \mathrm{e}-5$ & $5.8 \mathrm{e}-10$ & $9.0 \mathrm{e}-17$ & $4.3 \mathrm{e}-29$ & $1.2 \mathrm{e}-49$ & $1.1 \mathrm{e}-86$ \\
\hline 5 & $1.8 \mathrm{e}-6$ & $5.2 \mathrm{e}-11$ & $6.5 \mathrm{e}-18$ & $1.3 \mathrm{e}-30$ & $1.0 \mathrm{e}-53$ & $3.7 \mathrm{e}-93$ \\
\hline \multicolumn{7}{|c}{ TABLE } \\
\hline \multicolumn{7}{l}{}
\end{tabular}

MINIMAL THROUGHPUT FOR EACH $\mathrm{SF}_{m}$ [KBIT/S ]

Table I based on their distance $r_{n}$ : device $n$ uses $\mathrm{SF}_{m}$ if $r_{n} \in\left(l_{m-1}, l_{m}\right]$.

\section{Choice of $\mathrm{N}_{\max }$ given a target minimum throughput}

To determine the quota of each SF, we fix a target minimal throughput equal to 1 [bit/s]. We have run preliminary simulations over 100000 frames. Table II represents the minimal short-term average rate achieved on each SF, for different values of $\mathrm{N}_{\max }$. We can observe that to guarantee the target minimal throughput of 1 [bit/s], we can have at most three devices assigned to $\mathrm{SF}_{7}$ but only one device for the other SFs. In the sequel, the proposed method is compared to the baseline SF-allocation schemes. For fair comparison, the number of simultaneously transmitting devices $A$ is equal to $\sum_{m \in \mathcal{M}} \mathrm{N}_{\max }(m)=8$ in each allocation period.

\section{Simulation results}

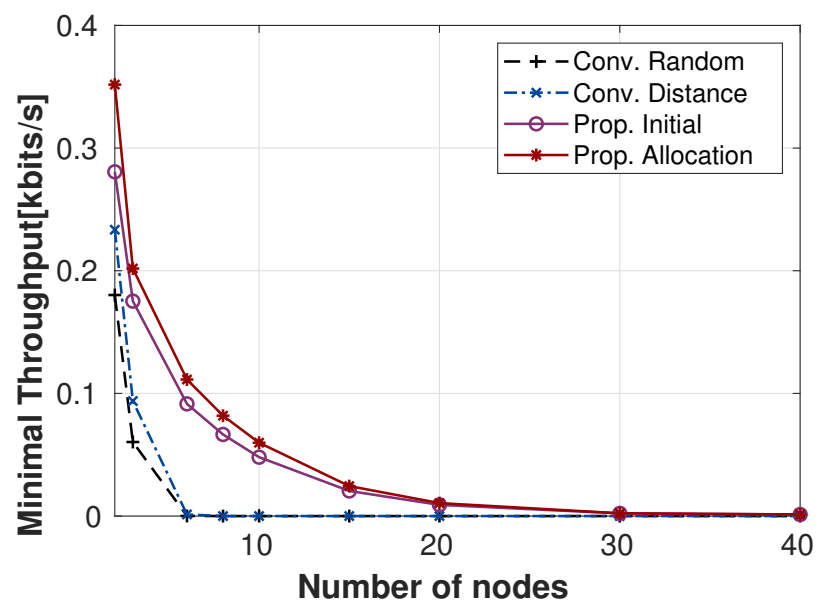

Fig. 2. Minimal short-term average rates, proposed and baseline algorithms

Figure 2 depicts the performance comparison of our SFallocation algorithm, and the baseline schemes in terms of minimal short-term average rates, against a varying number of end-devices. First, we can observe that the proposed allocation outperforms both the conventional random and distance allocations for all values of $N$. While baseline schemes lead to an early drop of minimal rate (almost null for $N>6$ ), the proposed algorithms still provide a good minimal throughput for a much higher number of devices. We can also notice that our Matching Refinement (Prop. Allocation) provides a better minimal throughput than the Initial Matching (Prop. Initial).

Next, Figure 3 shows the impact of maximizing the minimal

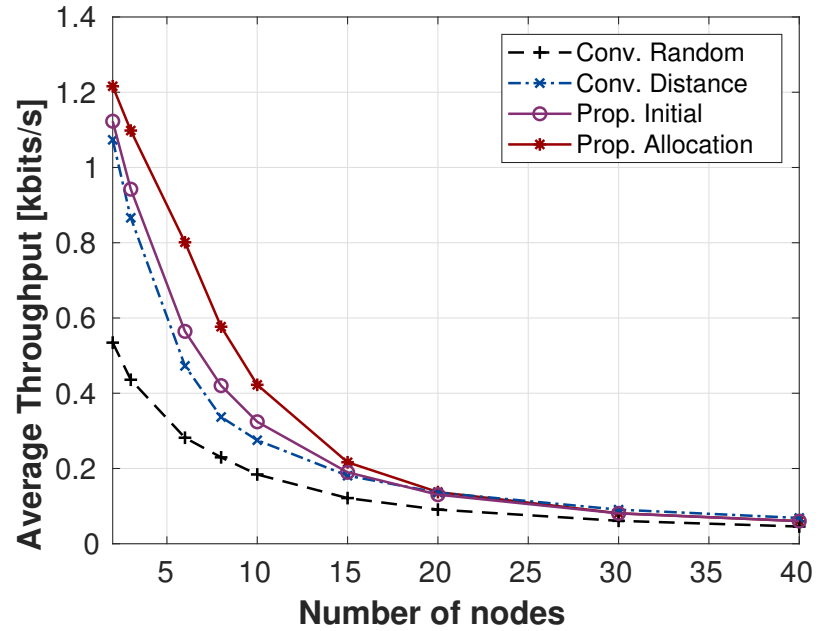

Fig. 3. Average network throughput, proposed and baseline algorithms

short-term average rates on the average network throughput over all end-devices. It can be clearly seen that the highest average network throughput is achieved by our proposed SFallocation method. Also, it can be noted that our Matching Refinement provides a significant improvement compared to the Initial Matching.

Finally, we evaluate the fairness levels of the different algo-

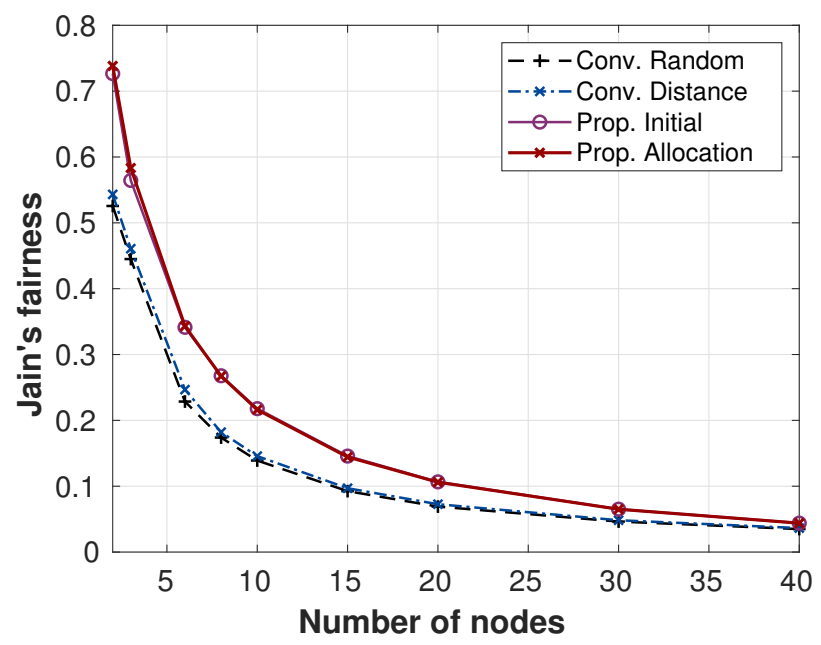

Fig. 4. Jain's fairness metric, proposed and baseline algorithms

rithms by the Jain's fairness index, given by $\mathcal{J}=\frac{\left(\sum_{n \in \mathcal{N}} U_{n}\right)^{2}}{N \times \sum_{n \in \mathcal{N}} U_{n}^{2}}$.

Figure 4 shows that by the considered max-min strategy and matching-based methodology, the proposed algorithms significantly enhance the system fairness level compared to baseline methods.

Overall, the proposed SF-allocation method provides remarkable performance improvements, jointly in terms of minimal achievable rates, network throughput and fairness, with a 
limited computational complexity suitable for LoRa gateways.

\section{CONCLUSION}

We have considered the problem of fairness-aware SFallocation in a LoRa-based network composed of IoT enddevices. Unlike most previous works, we have integrated both the effects of co-SF and inter-SF interferences in the expression of the achievable uplink short-term average rate of each end-device. We have then formulated our SF allocation problem as a max-min optimization of the uplink short-term average rate of end-devices. To solve this NP-hard optimization problem, we have proposed an SF-allocation algorithm based on matching theory, and proved its stability, convergence properties and its reasonable complexity. Numerical results show that our SF-allocation strategy not only outperforms the conventional algorithms in terms of minimal rate, but also jointly in terms of network throughput and fairness. For future work, this problem will be extended by jointly controlling the transmission power and SF allocation and by designing distributed algorithms. Energy efficiency aspects will be also investigated.

\section{REFERENCES}

[1] LoRa Alliance. LoRaWAN - What is it?, A technical overview of LoRa and LoRaWAN? [Online]. Available: www.lora-alliance.org

[2] J. Haxhibeqiri, F. Van den Abeele, I. Moerman and J. Hoebeke, "LoRa Scalability: A Simulation Model Based on Interference Measurments," in Sensors, May 2017, pp. 1-7.

[3] Semtech Corporation. (2013) LoRa Modem Design Guide, SX1272/3/6/7/8. [Online]. Available: www.semtech.com

[4] LoRa Alliance. LoRa Alliance LoRaWAN ${ }^{\mathrm{TM}} 1.1$ Specification. [Online]. Available: https://lora-alliance.org/sites/default/files/2018-04/ lorawantm_specification_-v1.1.pdf

[5] D. Corce, M. Gucciardo, I. Tinnirello, D. Garlisi, S. Mangione, "Impact of Spreading Factor Imperfect Orthogonality in LoRa Communications," in Springer, Towards a Smart and Secure Future Internet, vol. 766, Sep 2017, pp. 6510-6523.

[6] G. Zhu, C. Liao, M. Suzuki, Y. Narusue, H. Morikawa, "Evaluation of LoRa receiver performance under co-technology interference," in IEEE CCNC, Mar 2018.

[7] A. Waret, M. Kaneko, A. Guitton, N. El Rachkidy, "LoRa Throughput Analysis with Imperfect Spreading Factor Orthogonality," in IEEE Wireless Communication Letters, in press., 2018.

[8] Z. Qin and J. A. McCann, "Resource Efficiency in Low-Power WideArea Networks for IoT Applications," in Proc. IEEE Global Commun. Conf. (GLOBECOM), Dec 2017, pp. 1-7.

[9] B. Reynders, W. Meert and S. Pollin, "Power and Spreading Factor Control in Low Power Wide Area Networks," in IEEE ICC Symposium Internet of Things Track, Jul 2017, pp. 1-5.

[10] N. El Rachkidy, A. Guitton, M. Kaneko, "Decoding Superposed LoRa Signals," in the 43rd IEEE Conf. on Local Computer Networks (IEEE LCN 2018), Chicago, USA, Oct 2018.

[11] M. Bor, U. Roedig, T. Voigt and J.M. Alonso, "Do LoRa Low-Power Wide-Area Networks Scale," in ACM MSWiM, Malta, Nov 2016.

[12] O. Georgiou and U. Raza, "Low Power Wide Area Network Analysis: Can LoRa Scale?" in IEEE Wireless Communications Letters, vol. 6, Apr 2017, pp. 162 - 165.

[13] S. Boyd and L. Vandenberghe, Convex Optimization. Cambridge University Press, 2004.

[14] Y. Gu, W. Saad, M. Bennis, M. Debbah, and Z. Han, "Matching Theory for Future Wireless Networks: Fundamentals and Applications," in IEEE Communications Magazine, vol. 53, May 2015, pp. 52 - 59.

\section{APPENDIX}

We now prove the expression (8). Using the random instantaneous SNR variables $\gamma_{n m}$ for all $(n, m)$, (7) can be developed as

$$
\begin{aligned}
& P\left(\frac{\gamma_{n m}}{\sum_{\substack{(i, j) \in \in_{-m} \\
\mathcal{N}_{-n} \times \mathcal{M}}} s_{i j} \gamma_{i j}+1} \geq \tilde{\theta}_{m} \mid \gamma_{11}, \ldots \gamma_{n-1 m-1}, \gamma_{n+1 m+1}, \ldots \gamma_{N M}\right) \\
& \times P\left(\gamma_{11}, \ldots \gamma_{n-1 m-1}, \gamma_{n+1 m+1}, \ldots \gamma_{N M}\right) .
\end{aligned}
$$

The variables $\gamma_{n m}$ are modeled as exponential random variables with mean $\bar{\gamma}_{n m}$. Therefore, by marginalizing over these variables we obtain

$$
\begin{aligned}
& \int_{11} \cdots \int_{\gamma_{n-1 m-1}} \int_{\gamma_{n+1 m+1}} \cdots \int_{\gamma_{N M}} P \underbrace{\left(\gamma_{n m} \geq \tilde{\theta}_{m}\left(\sum_{\mathcal{N}_{-n}^{(i, j) \in} \times \mathcal{M}_{-m}} s_{i j} \gamma_{i j}+1\right)\right)}_{A} \\
& \times P\left(\gamma_{11}, \ldots \gamma_{n-1 m-1}, \gamma_{n+1 m+1}, \ldots \gamma_{N M}\right) \mathrm{d} \gamma_{11} \ldots \mathrm{d} \gamma_{N M} .
\end{aligned}
$$

First, we calculate $A$ where $p_{\gamma}\left(\gamma_{n m}\right)=\frac{1}{\bar{\gamma}_{n m}} \mathrm{e}^{-\frac{\gamma_{n m}}{\bar{\gamma}_{n m}}}$

$$
\begin{aligned}
A & =\int^{\infty} \tilde{\theta}_{m}\left(\sum_{\left.(i, j) \in \mathcal{N}_{-n} \times \mathcal{M}_{-m} s_{i j} \gamma_{i j}+1\right)} p_{\gamma}\left(\gamma_{n m}\right) \mathrm{d} \gamma_{n m}\right. \\
& =\mathrm{e}^{-\frac{\tilde{\theta}_{m}}{\bar{\gamma}_{n m}}} \prod_{(i, j) \in \mathcal{N}_{-n} \times \mathcal{M}_{-m}} \frac{1}{\frac{\tilde{\theta}_{m} s_{i j} \bar{\gamma}_{i j}}{\bar{\gamma}_{n m}}+1} .
\end{aligned}
$$

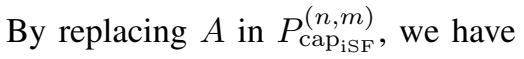

$$
\begin{aligned}
& P_{\text {capisf }}^{(n, m)}=\mathrm{e}^{-\frac{\tilde{\theta}_{m}}{\bar{\gamma}_{n m}}} \prod_{\substack{(i, j) \in \\
\mathcal{N}_{-n} \times \mathcal{M}_{-m}}} \int_{\gamma_{i j}} \mathrm{e}^{-\frac{\tilde{\theta}_{m} s_{i j} \gamma_{i j}}{\bar{\gamma}_{n m}}} p_{\gamma}\left(\gamma_{i j}\right) \mathrm{d} \gamma_{i j} \\
& =\mathrm{e}^{-\frac{\tilde{\theta}_{m}}{\bar{\gamma}_{n m}}} \prod_{\substack{(i, j) \in \\
\mathcal{N}_{-n} \times \mathcal{M}_{-m}}} \frac{1}{\frac{\tilde{\theta}_{m} s_{i j} \bar{\gamma}_{i j}}{\bar{\gamma}_{n m}}+1} .
\end{aligned}
$$

Finally, we obtain

$$
P_{\text {cap }_{i \text { SF }}}^{(n, m)}=\mathrm{e}^{-\frac{\tilde{\theta}_{m} \sigma_{c}^{2} r_{n}^{\alpha}}{\mathrm{A}\left(f_{c}\right) P_{\max }}} \prod_{\substack{(i, j) \in \\ \mathcal{N}_{-n} \times \mathcal{M}_{-m}}} \frac{1}{\tilde{\theta}_{m} s_{i j}\left(\frac{r_{n}}{r_{i}}\right)^{\alpha}+1} .
$$

